\title{
Photon propagation in a cold axion background with and without magnetic field
}

\author{
D. Espriu and A. Renau \\ Departament d'Estructura i Constituents de la Matèria and Institut de Ciències del Cosmos (ICCUB), \\ Universitat de Barcelona, Martí i Franquès 1, 08028 Barcelona, Spain
}

(Received 8 July 2011; published 18 January 2012)

\begin{abstract}
A cold relic axion condensate resulting from vacuum misalignment in the early Universe oscillates with a frequency $m$, where $m$ is the axion mass. We determine the properties of photons propagating in a simplified version of such a background where the sinusoidal variation is replaced by a square wave profile. We prove that previous results, which indicated that charged particles moving fast in such a background radiate (originally derived assuming that all momenta involved were much larger than $m$ ), hold for long wavelengths, too. We also analyze in detail how the introduction of a magnetic field changes the properties of photon propagation in such a medium. We briefly comment on possible astrophysical implications of these results.
\end{abstract}

DOI: 10.1103/PhysRevD.85.025010

PACS numbers: 14.80.Va, 96.50.S-, 95.35.+d

\section{INTRODUCTION}

Axions, originally introduced to solve the strong $C P$ problem [1], are to this date a viable candidate to constitute the dark matter of the Universe [2]. Their contribution to the mass density results from the energy stored in the collective oscillations around the minimum of the axion potential

$$
a(t)=a_{0} \cos (m t),
$$

with a frequency that is given by the axion mass $m$. We know that this mass must be somewhere in the range [3]

$$
1 \mathrm{eV}>m>10^{-6} \mathrm{eV}
$$

The coupling of axions to photons takes place through the universal term ${ }^{1}$

$$
\mathcal{L}_{a \gamma \gamma}=g_{a \gamma \gamma} \frac{\alpha}{2 \pi} \frac{a}{f_{a}} F_{\mu \nu} \tilde{F}^{\mu \nu},
$$

where $\tilde{F}^{\mu \nu}=\frac{1}{2} \epsilon^{\mu \nu \alpha \beta} F_{\alpha \beta}$ is the dual electromagnetic tensor. The dimensionful quantity $f_{a}$ is the axion decay constant - the equivalent of $f_{\pi}$ as axions are assumed to be the pseudo-Goldstone bosons associated with the breaking of the Peccei-Quinn symmetry $U_{\mathrm{PQ}}(1)$ [1]. On $f_{a}$, we have a range of bounds: $f_{a}>10^{4} \mathrm{GeV}$ coming from direct experimental searches of axions coupling directly to matter [5]; $f_{a}>10^{7} \mathrm{GeV}$ from (somewhat weaker) astrophysical constraints [6], largely mass independent; or $f_{a}>10^{7}$ for $0.02 \mathrm{eV}<m<0.4 \mathrm{eV}$ coming from phase II of the CAST experiment [7]. For some reviews of the experimental/ observational search for axions, see Ref. [3].

\footnotetext{
${ }^{1}$ This term is often written as $\mathcal{L}_{A \gamma \gamma}=\frac{G_{A \gamma \gamma}}{4} F_{\mu \nu} \tilde{F}^{\mu \nu} \phi_{A}$, where $\phi_{A}$ is the axion field [4]. Both $G_{A \gamma \gamma}$ and $g_{a \gamma \gamma}$ are used interchangeably in the axion literature as coupling constants having dimensions $E^{-1}$. The constant $g_{a \gamma \gamma}$ used here is, however, dimensionless, and it should not be confused with the latter.
}

The constant $g_{a \gamma \gamma}$ is model-dependent, but it is typically of order 1 in most axion models [8]. The axion, being a pseudo-Goldstone boson, satisfies the relation $f_{a} m \simeq$ constant $\simeq f_{\pi} m_{\pi}$, thus constraining the basic parameters of the theory. However, the results presented below apply also to other light pseudoscalar particles, sometimes termed axionlike particles (ALP). The coupling between ALP and photons could in principle be stronger, since it is not related to their mass.

Integrating by parts, we can write the term coupling axions or ALP to photons like

$$
\mathcal{L}_{a \gamma \gamma}=\frac{1}{2} \eta_{\mu} A_{\nu} \tilde{F}^{\mu \nu},
$$

with

$$
\eta_{\mu}=\eta(t) \delta_{\mu 0}, \quad \eta(t)=\eta_{0} \sin m t .
$$

The Lagrangian for a photon in the cold axion background is then

$$
\mathcal{L}=-\frac{1}{4} F^{\mu \nu} F_{\mu \nu}+\frac{1}{2} \eta_{\mu} A_{\nu} \tilde{F}^{\mu \nu},
$$

and the relevant quantity to determine the physical effect of this coupling is

$$
\eta_{0}=2 g_{a \gamma \gamma} \frac{\alpha}{\pi} \frac{a_{0} m}{f_{a}} .
$$

Now, we can proceed to quantizing the photon field in such a background. This has been previously done in Ref. [9] in the case where $\eta(t)$ is assumed to be a constant, $\eta(t)=\eta_{0}$. It was found that in this case, the two physical photon polarizations get their dispersion relations modified in the following way:

$$
\omega_{ \pm}=\sqrt{\vec{k}^{2} \pm \eta_{0}|\vec{k}|} .
$$

As a consequence, processes that are forbidden on Lorentzinvariance grounds, such as $\gamma \rightarrow e^{+} e^{-}$or $e \rightarrow e \gamma$, have a nonvanishing probability if certain kinematical constraints are fulfilled. The interested reader can see Ref. [10] for 
possible observable consequences. If measured, these effects would constitute prima facie evidence that not only axions or ALP exist but they do constitute the primary ingredient of the dark matter of the Universe.

It was argued in Refs. [10,11] that taking $\eta(t)$ as a constant was a good approximation if the momenta of all particles involved in the process were larger than $m$, the period of oscillations. However, if the wavelength of some of the particles are comparable or lower than the period of oscillation, one must necessarily deal with a timedependent external potential. Thus, it seems to us quite important to establish the basic principles of photon propagation in a time-dependent axion background. For this reason, in this paper, we solve the problem of photon propagation in an oscillatory, but spatially constant, axion background exactly. We shall also include an external magnetic field to see how the combined effect modifies the properties of photons moving in such an environment. We will discuss at the end of the paper some possible physical consequences.

To keep the paper technically simple, we have approximated the sinusoidal time dependence of the background by a square wave with the same period. A sinusoidal wave involves Mathieu special functions complicating the calculation enormously. We base this approximation on the similarity of the present effect with the emergence of the band structure in periodic potentials [12], exchanging time and space and momenta and energies. It is well-known in solid state physics that even such a simple model fully captures the essentials of metallic conductors and semiconductors. Therefore, we firmly believe that the physics of the problem being discussed remains unaltered by our technical simplification.

\section{SOLVING FOR THE EIGENMODES AND EIGENVALUES}

We introduce a Fourier transform with respect to the spatial coordinates only and write the photon field as

$$
A_{\mu}(t, \vec{x})=\int \frac{d^{3} k}{(2 \pi)^{3}} e^{i \vec{k} \cdot \vec{x}} \hat{A}_{\mu}(t, \vec{k}) .
$$

The equation for $\hat{A}_{\nu}(t, \vec{k})$ is

$$
\left[g^{\mu \nu}\left(\partial_{t}^{2}+\vec{k}^{2}\right)-i \epsilon^{\mu \nu \alpha \beta} \eta_{\alpha} k_{\beta}\right] \hat{A}_{\nu}(t, \vec{k})=0 .
$$

We now define

$$
S_{\lambda}^{\nu}=\epsilon^{\mu \nu \alpha \beta} \eta_{\alpha} k_{\beta} \epsilon_{\mu \lambda \rho \sigma} \eta^{\rho} k^{\sigma},
$$

which can also be written as

$$
\begin{aligned}
S^{\mu \nu}= & {\left[(\eta \cdot k)^{2}-\eta^{2} k^{2}\right] g^{\mu \nu}+k^{2} \eta^{\mu} \eta^{\nu} } \\
& +\eta^{2} k^{\mu} k^{\nu}-(\eta \cdot k)\left(\eta^{\mu} k^{\nu}+\eta^{\nu} k^{\mu}\right),
\end{aligned}
$$

and

$$
P_{ \pm}^{\mu \nu}=\frac{S^{\mu \nu}}{S} \mp \frac{i}{\sqrt{2 S}} \epsilon^{\mu \nu \alpha \beta} \eta_{\alpha} k_{\beta}, \quad S=S_{\mu}^{\mu}=2 \eta^{2} \vec{k}^{2} .
$$

The properties of these quantities are discussed in Ref. [9]. Note that the time dependence [due to $\eta(t)]$ in $P_{ \pm}^{\mu \nu}$ cancels. With the help of these projectors, we can write Eq. (10) as

$$
\left[g^{\mu \nu}\left(\partial_{t}^{2}+\vec{k}^{2}\right)+\sqrt{\frac{S}{2}}\left(P_{+}^{\mu \nu}-P_{-}^{\mu \nu}\right)\right] \hat{A}_{\nu}(t, \vec{k})=0 .
$$

To solve the equations of motion, we introduce the polarization vectors defined in Ref. [9] and write ${ }^{2}$

$$
\hat{A}_{\nu}(t, \vec{k})=\sum_{\lambda=+,-} f_{\lambda}(t) \varepsilon_{\nu}(\vec{k}, \lambda)
$$

These vectors satisfy

$$
P_{ \pm}^{\mu \nu} \varepsilon_{\nu}(\vec{k}, \pm)=\varepsilon^{\mu}(\vec{k}, \pm), \quad P_{ \pm}^{\mu \nu} \varepsilon_{\nu}(\vec{k}, \mp)=0
$$

and do not depend on $t$, so

$$
\left[\partial_{t}^{2}+\vec{k}^{2} \pm \eta(t)|\vec{k}|\right] f_{ \pm}(t)=0 .
$$

As mentioned, we will approximate the sine function in $\eta(t)$ by a square wave function:

$$
\eta(t)=\left\{\begin{array}{ll}
+\eta_{0} & 2 n T<t<(2 n+1) T \\
-\eta_{0} & (2 n+1) T<t<2 n T
\end{array} .\right.
$$

The relevant parameters are

$$
\eta_{0}=2 g_{a \gamma \gamma} \frac{\alpha}{\pi} \frac{a_{0} m}{f_{a}}, \quad T=\frac{\pi}{m} .
$$

There is an equation for each polarization. However, they are related. To recover one from the other, we can just make the replacement $\eta_{0} \rightarrow-\eta_{0}$. Also, because $\eta(t)$ changes sign after a time $T$ in the square wave approximation, one solution is a time-shifted copy of the other: $f_{-}(t)=f_{+}(t+T)$. In what follows, we will work in the case $\lambda=+$. It is obvious that the conclusions also apply to the other physical polarization, $\lambda=-$.

Since $\eta(t)$ is piecewise-defined, we will solve the equation in two regions:

(i) Region 1: $0<t<T, \eta(t)=\eta_{0}$

$$
\begin{gathered}
\frac{d^{2} f_{1}(t)}{d t^{2}}+\left(\vec{k}^{2}+\eta_{0}|\vec{k}|\right) f_{1}(t)=0, \\
f_{1}(t)=A^{\prime} e^{i \alpha t}+A e^{-i \alpha t}, \quad \alpha^{2}=\vec{k}^{2}+\eta_{0}|\vec{k}| .
\end{gathered}
$$

\footnotetext{
${ }^{2}$ When $\eta_{\mu}$ only has a temporal component, these polarization vectors actually reduce to the usual ones.
} 
(ii) Region 2: $-T<t<0, \eta(t)=-\eta_{0}$

$$
\begin{gathered}
\frac{d^{2} f_{2}(t)}{d t^{2}}+\left(\vec{k}^{2}-\eta_{0}|\vec{k}|\right) f_{2}(t)=0, \quad \\
f_{2}(t)=B^{\prime} e^{i \beta t}+B e^{-i \beta t}, \quad \beta^{2}=\vec{k}^{2}-\eta_{0}|\vec{k}| .
\end{gathered}
$$

We impose that both functions coincide at $t=0$, and we do the same for their derivatives

$$
f_{1}(0)=f_{2}(0), \quad f_{1}^{\prime}(0)=f_{2}^{\prime}(0) .
$$

We now write $f(t)=e^{-i \omega t} g(t)$ and demand that $g(t)$ have the same periodicity as $\eta(t)$ :

$$
\begin{aligned}
& g_{1}(t)=e^{i \omega t} f_{1}(t)=A^{\prime} e^{i(\omega+\alpha) t}+A e^{i(\omega-\alpha) t}, \\
& g_{2}(t)=e^{i \omega t} f_{2}(t)=B^{\prime} e^{i(\omega+\beta) t}+B e^{i(\omega-\beta) t}, \\
& g_{1}(T)=g_{2}(-T), \quad g_{1}^{\prime}(T)=g_{2}^{\prime}(-T) .
\end{aligned}
$$

For these conditions to be fulfilled, the coefficients have to solve the linear system

$$
\begin{gathered}
A^{\prime}+A=B^{\prime}+B, \quad \alpha A^{\prime}-\alpha A=\beta B^{\prime}-\beta B, \\
e^{i(\omega+\alpha) T} A^{\prime}+e^{i(\omega-\alpha) T} A e^{-i(\omega+\beta) T} B^{\prime}+e^{-i(\omega-\beta) T} B, \\
(\omega+\alpha) e^{i(\omega+\alpha) T} A^{\prime}+(\omega-\alpha) e^{i(\omega-\alpha) T} A \\
=(\omega+\beta) e^{-i(\omega+\beta) T} B^{\prime}+(\omega-\beta) e^{-i(\omega-\beta) T} .
\end{gathered}
$$

The linear system can be expressed as

$$
\hat{M}\left(\begin{array}{c}
A^{\prime} \\
A \\
B^{\prime} \\
B
\end{array}\right)=\left(\begin{array}{l}
0 \\
0 \\
0 \\
0
\end{array}\right)
$$

with

$$
\hat{M}^{T}=\left(\begin{array}{cccc}
1 & \alpha & e^{i(\omega+\alpha) T} & (\omega+\alpha) e^{i(\omega+\alpha) T} \\
1 & -\alpha & e^{i(\omega-\alpha) T} & (\omega-\alpha) e^{i(\omega-\alpha) T} \\
-1 & -\beta & -e^{-i(\omega+\beta) T} & -(\omega+\beta) e^{-i(\omega+\beta) T} \\
-1 & \beta & -e^{-i(\omega-\beta) T} & -(\omega-\beta) e^{-i(\omega-\beta) T}
\end{array}\right) .
$$

The problem being discussed here is formally similar to the solution of the Kronig-Penney [12] one-dimensional periodic potential, except the periodicity is now in time rather than in space.

In order to find a nontrivial solution, one has to demand the condition of vanishing determinant of $\hat{M}$, which is

$$
\begin{aligned}
\cos (2 \omega T)= & \cos (\alpha T) \cos (\beta T) \\
& -\frac{\alpha^{2}+\beta^{2}}{2 \alpha \beta} \sin (\alpha T) \sin (\beta T),
\end{aligned}
$$

with $\alpha$ and $\beta$ given by Eqs. (22) and (24), respectively. In order to get analytical expressions, we will work in the limit of long wavelengths $|\vec{k}| T \ll 1$, which is just the one that is potentially problematic as discussed in the introduction. Expanding both sides,

$$
\omega^{2}-\frac{1}{3} \omega^{4} T^{2}+\ldots=\vec{k}^{2}-\left(\frac{1}{3} \vec{k}^{4}-\frac{1}{12} \eta_{0}^{2} \vec{k}^{2}\right) T^{2}+\ldots,
$$

which means

$$
\omega^{2} \approx\left(1+\frac{\eta_{0}^{2} T^{2}}{12}\right) \vec{k}^{2}
$$

If the determinant vanishes, the system to solve is

$$
\left(\begin{array}{ccc}
1 & 1 & -1 \\
0 & 1 & -\frac{1}{2}\left(1-\frac{\beta}{\alpha}\right) \\
0 & 0 & 1
\end{array}\right)\left(\begin{array}{c}
A^{\prime} \\
A \\
B^{\prime}
\end{array}\right)=\left(\begin{array}{c}
1 \\
\frac{1}{2}\left(1+\frac{\beta}{\alpha}\right) \\
h(\alpha, \beta, T)
\end{array}\right) B,
$$

where

$$
h(\alpha, \beta, T)=-\frac{\alpha-\beta}{\alpha+\beta} \frac{e^{i \alpha T}-e^{-i 2 \omega T} e^{i \beta T}}{e^{i \alpha T}-e^{-i 2 \omega T} e^{-i \beta T}},
$$

leading to

$$
\begin{aligned}
\frac{A^{\prime}}{B}= & {\left[1-\frac{\alpha-\beta}{\alpha+\beta} \frac{e^{i \alpha T} e^{i 2 \omega T}-e^{i \beta T}}{e^{i \alpha T} e^{i 2 \omega T}-e^{-i \beta T}}-\frac{1}{2}\left(1+\frac{\beta}{\alpha}\right)\right.} \\
& \left.+\frac{1}{2}\left(1-\frac{\beta}{\alpha}\right) \frac{\alpha-\beta}{\alpha+\beta} \frac{e^{i \alpha T} e^{i 2 \omega T}-e^{i \beta T}}{e^{i \alpha T} e^{i 2 \omega T}-e^{-i \beta T}}\right] \\
\frac{A}{B}= & {\left[\frac{1}{2}\left(1+\frac{\beta}{\alpha}\right)-\frac{1}{2}\left(1-\frac{\beta}{\alpha}\right) \frac{\alpha-\beta}{\alpha+\beta}\right.} \\
& \left.\times \frac{e^{i \alpha T} e^{i 2 \omega T}-e^{i \beta T}}{e^{i \alpha T} e^{i 2 \omega T}-e^{-i \beta T}}\right] \\
\frac{B^{\prime}}{B}= & {\left[-\frac{\alpha-\beta}{\alpha+\beta} \frac{e^{i \alpha T} e^{i 2 \omega T}-e^{i \beta T} e^{i 2 \omega T}-e^{-i \beta T}}{e^{i \beta T}}\right] }
\end{aligned}
$$

In the limit $\eta_{0} \ll|\vec{k}|,|\vec{k}| T \ll 1$,

$$
\frac{A^{\prime}}{B} \approx-\frac{B^{\prime}}{B} \approx \frac{1}{4} \frac{\eta_{0}}{|\vec{k}|}, \quad \frac{A}{B} \approx 1-\frac{\eta_{0}}{2|\vec{k}|} .
$$

Finally, imposing the usual normalization,

$$
\int f_{k}(t) f_{k^{\prime}}^{*}(t)=2 \pi \delta\left(|\vec{k}|-\left|\vec{k}^{\prime}\right|\right)
$$

we get 


$$
\begin{aligned}
B= & {\left[\frac{\sqrt{\vec{k}^{2}+\eta_{0}|\vec{k}|}}{2|\vec{k}|+\eta_{0}}\left(\left|\frac{A}{B}\right|^{2}+\left|\frac{A^{\prime}}{B}\right|^{2}\right)\right.} \\
& \left.+\frac{\sqrt{\vec{k}^{2}-\eta_{0}|\vec{k}|}}{2|\vec{k}|-\eta_{0}}\left(1+\left|\frac{B^{\prime}}{B}\right|^{2}\right)\right]^{-1 / 2} \\
\approx & \left(1+\frac{\eta_{0}}{4|\vec{k}|}\right) .
\end{aligned}
$$

This completes the determination of the eigenvectors.

\section{A. Exact determination of the eigenvalues}

We can also solve Eq. (30) exactly, without having to assume the long-wavelength limit as above, but this can be done only numerically. The solution only depends on $\eta_{0}$ and $m$ through the dimensionless combination $\eta_{0} T$. There are values of $k$ for which there is no solution, as seen in Fig. 1. However, these gaps get narrower when the product $\eta_{0} T$ decreases. In practice, the largest possible physical value for this quantity is $\eta_{0} T=10^{-14}$, and then the gaps are practically nonexistent and certainly totally irrelevant for the purposes of the present paper.

It is interesting to investigate whether complex solutions exist for $\omega$ in the forbidden narrow bands. We note that the right-hand side of Eq. (30) is necessarily real, thus $\omega$ must be purely real or purely imaginary. In the latter case, the left-hand side is replaced by a cosh, having as argument the imaginary part of $2 \omega T$. For this to have a solution, the right-hand side must be positive and larger than 1 . Inspection of this term reveals that it is larger than one in

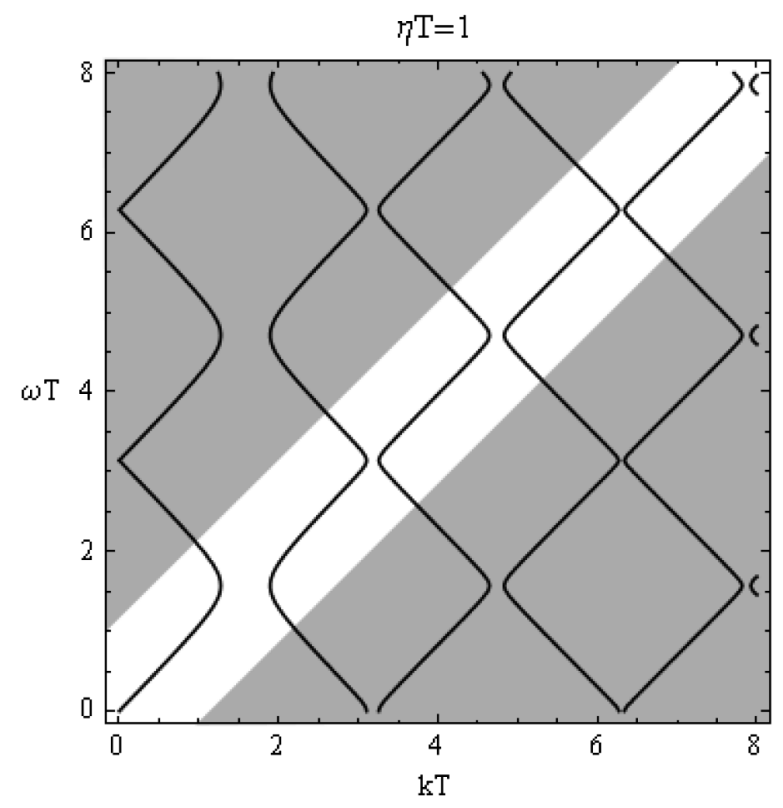

the forbidden zones but actually alternates sign. Therefore, not even an imaginary solution exists for the first, third, etc., forbidden regions.

\section{B. Calculation of the transition $e \rightarrow e \gamma$}

In order to make the photon field Hermitian, we add Eq. (9) and its conjugate. Introducing creation and annihilation operators for each one of the proper modes, we get (both polarizations are included)

$$
\begin{aligned}
A_{\mu}(t, \vec{x})= & \int \frac{d^{3} k}{(2 \pi)^{3}} \sum_{\lambda}\left[a(\vec{k}, \lambda) g(t, \vec{k}, \lambda) \varepsilon_{\mu}(\vec{k}, \lambda) e^{-i k x}\right. \\
& \left.+a^{\dagger}(\vec{k}, \lambda) g^{*}(t, \vec{k}, \lambda) \varepsilon_{\mu}^{*}(\vec{k}, \lambda) e^{i k x}\right]
\end{aligned}
$$

where $k x \equiv \omega t-\vec{k} \cdot \vec{x}$. Now, we want to compute $\langle f|S| i\rangle$ for an initial state of one electron of momentum $p$ and a final state of an electron of momentum $q$ and a photon of momentum $k=p-q$.

$$
\begin{aligned}
\langle f|S| i\rangle= & i e \varepsilon_{\mu}^{*}(\vec{k}, \lambda) \bar{u}_{q} \gamma^{\mu} u_{p}(2 \pi)^{3} \delta^{(3)}(\vec{k}+\vec{q}-\vec{p}) \\
& \times \int d t g^{*}(t, \vec{k}, \lambda) e^{i\left(\omega+E_{q}-E_{p}\right) t} .
\end{aligned}
$$

If we take $\eta(t)$ constant, $g(t, \vec{k}, \lambda)=1$, and we have

$$
\langle f|S| i\rangle=i e \varepsilon_{\mu}^{*}(\vec{k}, \lambda) \bar{u}_{q} \gamma^{\mu} u_{p}(2 \pi)^{4} \delta^{(4)}(k+q-p) .
$$

In the square wave approximation (19), the time integration yields

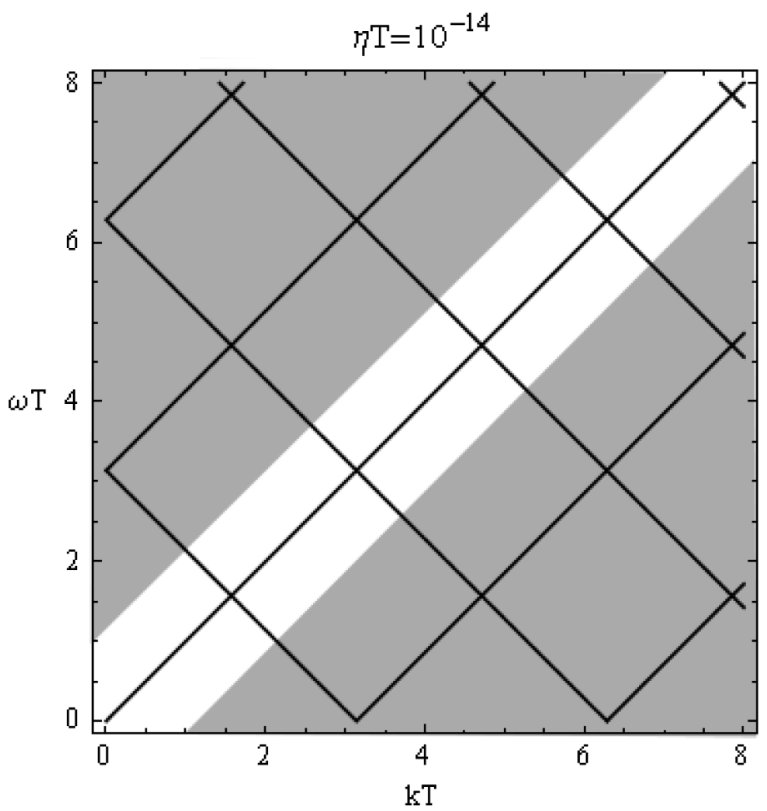

FIG. 1. Plots of the solution for $\eta_{0} T=1$ and $\eta_{0} T=10^{-14}$. In the $\eta_{0} T \rightarrow 0$ limit, the solutions correspond to the straight lines $\omega \sim|\vec{k}|$ (plus their periodic repetitions). Small gaps develop, but they become only physically significant when $\eta_{0} T=\mathcal{O}(1)$. The physical region corresponds to the white area; the gray areas are just periodic repetitions. 


$$
\begin{aligned}
\langle f|S| i\rangle= & i e \bar{u}_{q} \gamma^{\mu} u_{p} \varepsilon_{\mu}^{*}(\vec{k}, \lambda)(2 \pi)^{3} \delta(\vec{k}+\vec{q}-\vec{p}) \\
& \times \pi\left\{A \delta\left(\alpha+E_{q}-E_{p}\right)+B \delta\left(\beta+E_{q}-E_{p}\right)\right. \\
& \left.+A^{\prime} \delta\left(-\alpha+E_{q}-E_{p}\right)-B^{\prime} \delta\left(-\beta+E_{q}-E_{p}\right)\right\}
\end{aligned}
$$

$$
\begin{aligned}
& \approx i e \bar{u}_{q} \gamma^{\mu} u_{p} \varepsilon_{\mu}^{*}(\vec{k}, \lambda)\left(1+\frac{\eta_{0}}{4|\vec{k}|}\right)(2 \pi)^{3} \delta(\vec{k}+\vec{q}-\vec{p}) \\
& \quad \times \pi\left\{\left(1-\frac{\eta_{0}}{2|\vec{k}|}\right) \delta\left(\alpha+E_{q}-E_{p}\right)+\delta\left(\beta+E_{q}-E_{p}\right)\right. \\
& \left.\quad+\frac{\eta_{0}}{4|\vec{k}|}\left[\delta\left(-\alpha+E_{q}-E_{p}\right)+\delta\left(-\beta+E_{q}-E_{p}\right)\right]\right\} .
\end{aligned}
$$

Equation (42) holds for any value of $|\vec{k}|$. The $\approx$ symbol indicates the use of Eq. (36). It turns out that at the leading order in the $\eta_{0}$ expansion, this expression agrees exactly with the one obtained in Ref. [11] assuming that $\eta(t)$ was constant except for the fact that for each value of the polarization, only one of the two delta functions that are not suppressed by terms of the form $\eta_{0} /|\vec{k}|$ can be simultaneously satisfied; namely, the one that implies that $\alpha$ or $\beta$ equals $\sqrt{\vec{k}^{2}-\left|\eta_{0}\right||\vec{k}|}$, contributing with a factor $1 / 2$ with respect to what is found for constant $\eta$ to the amplitude. Thus, in the reduced matrix element $i \mathcal{M}$, one gets for each polarization exactly one-half of what is obtained if $\eta(t)$ is constant. But in the present case, both polarizations contribute, so finally we get $(1 / 2)^{2}+$ $(1 / 2)^{2}=1 / 2$ of the result obtained with constant $\eta(t)$.

As a consequence, the predictions concerning the radiation yield of a high-energy charged particle propagating in the cold axion background [10] are confirmed.

\section{PROPAGATION IN A MAGNETIC FIELD}

We will now compute the propagator of the photon field with two backgrounds: a cold axion background and a constant magnetic field. To do so, we take Eq. (3) and write the axion and photon fields as a background term plus a dynamical field. We get two relevant terms:

$$
\mathcal{L}_{a \gamma \gamma} \rightarrow \frac{1}{2} \epsilon^{\mu \nu \alpha \beta} \eta_{\mu} A_{\nu} \partial_{\alpha} A_{\beta}+\frac{2 g_{a \gamma \gamma} \alpha}{\pi f_{a}} a \partial_{\mu} A_{\nu} \tilde{F}^{\mu \nu},
$$

where $a$ is the axion field, $A_{\mu}$ is the photon field and $\tilde{F}^{\mu \nu}$ corresponds to a magnetic field: $\tilde{F}^{0 i}=B^{i}, \tilde{F}^{i j}=0$. The first term is just Eq. (4). Here, we will take $\eta(t)$ to be constant; therefore, the results that follow are valid only if the distance travelled by the photon, $l$, verifies $l<2 \pi / m$.

The vertices and Feynman rules corresponding to these terms are shown in Fig. 2. With the first vertex, we can compute the propagator in an axion background; see Fig. 3.

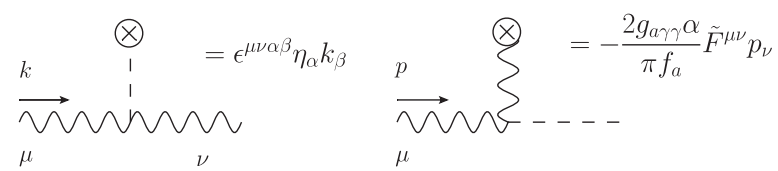

FIG. 2. The two relevant vertices. The corresponding Feynman rules are shown.

The successive interactions with the axion background can be summed up and the result is the propagator

$$
D^{\mu \nu}=-i\left(\frac{g^{\mu \nu}-X^{\mu \nu}}{k^{2}}+\frac{P_{+}^{\mu \nu}}{k^{2}-\eta_{0}|\vec{k}|}+\frac{P_{-}^{\mu \nu}}{k^{2}+\eta_{0}|\vec{k}|}\right) .
$$

The physical polarizations, projected out by $P_{ \pm}^{\mu \nu}$, exhibit poles at $\omega^{2}=\vec{k}^{2} \pm \eta_{0}|\vec{k}|$ as expected. The projectors are defined in Eq. (14) and $X^{\mu \nu}=\frac{S^{\mu \nu}}{\eta_{0}^{2}|\vec{k}|^{2}}$. Of course, the same result can be obtained by direct inversion of the photon equation of motion (10).

We now compute the propagator in the presence of a magnetic field, using the second term in Eq. (44). In order to do that, we use the propagator just found, represented by a double-wavy line, and include the interactions with the external magnetic field (see Fig. 4. The dashed line corresponds to the axion propagator. Summing all the diagrams, we get

$$
\mathcal{D}_{\mu \nu}=D_{\mu \nu}+f_{\mu} h_{\nu} \frac{-i g^{2}}{k^{2}-m^{2}+i g^{2} K}
$$

where

$$
\begin{gathered}
f_{\mu}=D_{\mu \alpha} \tilde{F}^{\alpha \lambda} k_{\lambda}, \quad h_{\nu}=\tilde{F}^{\sigma \phi} k_{\phi} D_{\sigma \nu}, \\
g=\frac{2 g_{a \gamma \gamma} \alpha}{\pi f_{a}}, \quad K=\tilde{F}^{\beta \rho} k_{\rho} D_{\beta \gamma} \tilde{F}^{\gamma \xi} k_{\xi} .
\end{gathered}
$$

In order to simplify the result, we shall assume that $\vec{k} \cdot \vec{B}=0$, which may correspond to an experimentally relevant situation. Then, we get

$$
\begin{aligned}
& f_{\mu}=i k_{0} g_{\mu}^{i} \frac{k^{2} B_{i}-i \eta_{0}(\vec{B} \times \vec{k})_{i}}{\left(k^{2}-\eta_{0}|\vec{k}|\right)\left(k^{2}+\eta_{0}|\vec{k}|\right)}, \\
& h_{\nu}=i k_{0} g_{\nu}^{j} \frac{k^{2} B_{j}+i \eta_{0}(\vec{B} \times \vec{k})_{j}}{\left(k^{2}-\eta_{0}|\vec{k}|\right)\left(k^{2}+\eta_{0}|\vec{k}|\right)},
\end{aligned}
$$

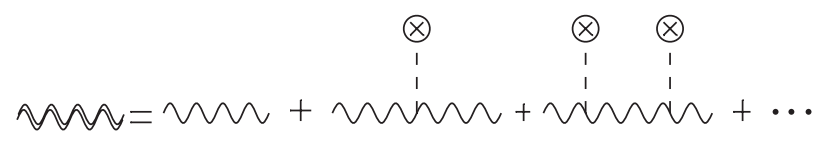

FIG. 3. Propagator in the axion background. 


$$
\text { man } \sum^{\otimes} \sum_{-m+\infty}^{\infty} m+\cdots
$$

FIG. 4. Full propagator after resummation of the interactions with the external $\vec{B}$ field.

$$
K=i k_{0}^{2} \vec{B}^{2} \frac{k^{2}}{\left(k^{2}-\eta_{0}|\vec{k}|\right)\left(k^{2}+\eta_{0}|\vec{k}|\right)},
$$

and, finally, defining $\vec{b} \equiv g \vec{B}$,

$$
\begin{aligned}
\mathcal{D}_{\mu \nu}= & D_{\mu \nu}+i k_{0}^{2} g_{\mu}^{j} g_{\nu}^{l}\left\{\frac{b_{j} b_{l}}{\left(k^{4}-\eta_{0}^{2} \vec{k}^{2}\right)\left(k^{2}-m^{2}\right)-k_{0}^{2} k^{2} \vec{b}^{2}}\right. \\
& \left.+\frac{i \eta_{0} k^{2}\left[b_{j}(\vec{b} \times \vec{k})_{l}-b_{l}(\vec{b} \times \vec{k})_{j}\right]-\eta_{0}^{2} \vec{b}^{2} \vec{k}^{2} X_{j l}}{\left(k^{4}-\eta_{0}^{2} \vec{k}^{2}\right)\left[\left(k^{4}-\eta_{0}^{2} \vec{k}^{2}\right)\left(k^{2}-m^{2}\right)-k_{0}^{2} k^{2} \vec{b}^{2}\right]}\right\} .
\end{aligned}
$$

\section{Particular case: no axion background}

As a relevant particular case, we now set $\eta_{0}=0$ in the previous expression, i.e. we consider only the influence of the magnetic background, and get

$$
\mathcal{D}_{\mu \nu}=D_{\mu \nu}+i k_{0}^{2} g_{\mu}^{j} g_{\nu}^{l} \frac{b_{j} b_{l}}{k^{4}\left(k^{2}-m^{2}\right)-k_{0}^{2} k^{2} \vec{b}^{2}},
$$

where now $D_{\mu \nu}$ stands for the usual photon propagator, obtained after setting $\eta_{0}=0$ in Eq. (45).

This propagator has poles when $k_{0}^{2}=\vec{k}^{2}$ and also for

$$
k_{0}^{2}=\frac{1}{2}\left(2 \vec{k}^{2}+m^{2}+\vec{b}^{2} \pm \sqrt{m^{4}+\vec{b}^{4}+2 m^{2} \vec{b}^{2}+4 \vec{b}^{2} \vec{k}^{2}}\right) .
$$

If we assume that $|\vec{b}|$ is a small parameter and expand in powers of it, these poles in the frequency plane lie at

$$
k_{0}^{2} \simeq \vec{k}^{2}\left(1+\frac{\vec{b}^{2}}{m^{2}}\right)+m^{2}+\vec{b}^{2}, \quad k_{0}^{2} \simeq \vec{k}^{2}\left(1-\frac{\vec{b}^{2}}{m^{2}}\right) .
$$

Physically, this pole structure corresponds to the perpendicular polarization vector $\epsilon_{\perp}$ propagating unchanged, while the parallel polarization $\epsilon_{\|}$and the would-be longitudinal polarization change their propagation. ${ }^{3}$

For completeness, we give the full propagator without the assumption $\vec{k} \cdot \vec{B}=0$ :

\footnotetext{
${ }^{3}$ The labels $\perp$ and $\|$ refer to directions perpendicular and parallel to the electric field, respectively, in the plane orthogonal to the propagation.
}

$$
\mathcal{D}_{\mu \nu}=D_{\mu \nu}+f_{\mu} h_{\nu} \frac{i}{k^{2}-m^{2}+K},
$$

where $D_{\mu \nu}=-i g_{\mu \nu} / k^{2}$ is the usual photon propagator and

$$
\begin{gathered}
f_{\mu}=h_{\mu}=\frac{1}{k^{2}}\left(g_{\mu 0} \vec{b} \cdot \vec{k}+g_{\mu j} b^{j} k_{0}\right), \\
K=\frac{1}{k^{2}}\left[(\vec{b} \cdot \vec{k})^{2}-\vec{b}^{2} k_{0}^{2}\right] .
\end{gathered}
$$

Let us now restore the condition $\vec{k} \cdot \vec{B}=0$ that is helpful in simplifying the formulae. In order to write the propagator in a more compact form, we introduce a four-vector $b^{\mu}=(0, \vec{b}=g \vec{B})$ :

$$
\mathcal{D}_{\mu \nu}(k)=\frac{-i g_{\mu \nu}}{k^{2}}+\frac{i k_{0}^{2} b_{\mu} b_{\nu}}{k^{2}\left[k^{2}\left(k^{2}-m^{2}\right)-k_{0}^{2} \vec{b}^{2}\right]} .
$$

Note the rather involved structure of the dispersion relation implied by Eq. (53). We consider the propagation of plane waves of well-defined frequency $\omega=k_{0}$ and moving in the $\hat{x}$ direction. The Fourier transform with respect to the spatial component will describe the space evolution of a photon state emitted at $x=0$ with polarization given by the vector $\epsilon_{0}$. We decompose

$$
\frac{1}{k^{2}\left[k^{2}\left(k^{2}-m^{2}\right)-k_{0}^{2} \vec{b}^{2}\right]}=\frac{A}{\vec{k}^{2}-k_{0}^{2}}+\frac{B}{\vec{k}^{2}-F}+\frac{C}{\vec{k}^{2}-G},
$$

where $F$ and $G$ are the roots of the denominator

$$
\begin{aligned}
& G=k_{0}^{2}-\frac{m^{2}}{2}-\frac{1}{2} \sqrt{m^{4}+4 k_{0}^{2} \vec{b}^{2}} \approx\left(1-\frac{\vec{b}^{2}}{m^{2}}\right) k_{0}^{2}-m^{2}, \\
& F=k_{0}^{2}-\frac{m^{2}}{2}+\frac{1}{2} \sqrt{m^{4}+4 k_{0}^{2} \vec{b}^{2}} \approx\left(1+\frac{\vec{b}^{2}}{m^{2}}\right) k_{0}^{2},
\end{aligned}
$$

in agreement with Eq. (55), and

$$
\begin{gathered}
A=\frac{1}{k_{0}^{2}-F} \frac{1}{k_{0}^{2}-G} \approx-\frac{1}{k_{0}^{2} \vec{b}^{2}}, \\
B=-\frac{1}{k_{0}^{2}-F} \frac{1}{F-G} \approx \frac{1}{k_{0}^{2} \vec{b}^{2}}, \\
C=\frac{1}{k_{0}^{2}-G} \frac{1}{F-G} \approx \frac{1}{m^{4}+3 k_{0}^{2} \vec{b}^{2}} \approx \frac{1}{m^{4}} .
\end{gathered}
$$

Even for the largest magnetic fields conceivable, the product $b=g B$ is rather small compared to the range of acceptable values of the axion mass, and it appears justified to neglect $C$. The space Fourier transform of the propagator is then 


$$
\begin{aligned}
\mathcal{D}_{\mu \nu}\left(k_{0}, x\right)= & -\frac{g_{\mu \nu}}{2 k_{0}} e^{i k_{0}|x|}-\frac{k_{0} b_{\mu} b_{\nu}}{2} A e^{i k_{0}|x|} \\
& -\frac{k_{0}^{2} b_{\mu} b_{\nu}}{2} \frac{B}{\sqrt{F}} e^{i \sqrt{F}|x|} .
\end{aligned}
$$

Let us now contract the propagator with the initial and final polarization vectors:

$$
\begin{aligned}
\epsilon^{\mu} \mathcal{D}_{\mu \nu}\left(k_{0}, x\right) \epsilon_{0}^{\nu} \approx & \frac{\overrightarrow{\boldsymbol{\epsilon}} \cdot \vec{\epsilon}_{0}}{2 k_{0}} e^{i k_{0}|x|}+\frac{(\overrightarrow{\boldsymbol{\epsilon}} \cdot \hat{b})\left(\vec{\epsilon}_{0} \cdot \hat{b}\right)}{2 k_{0}} e^{i k_{0}|x|} \\
& -\frac{(\overrightarrow{\boldsymbol{\epsilon}} \cdot \hat{b})\left(\overrightarrow{\boldsymbol{\epsilon}}_{0} \cdot \hat{b}\right)}{2 k_{0}} e^{i k_{0}|x|} e^{i\left(\vec{b}^{2} / 2 m^{2}\right) k_{0}|x|},
\end{aligned}
$$

where $\hat{b}=\vec{b} /|\vec{b}|$. Its squared modulus is

$$
\begin{aligned}
& \mid \epsilon^{\mu}\left.\mathcal{D}_{\mu \nu}\left(k_{0}, x\right) \epsilon_{0}^{\nu}\right|^{2} \\
& \quad=\frac{1}{4 k_{0}^{2}}\left[E_{1}^{2}+4\left(E_{1} E_{2}+E_{2}^{2}\right) \sin ^{2}\left(\frac{\vec{b}^{2}}{4 m^{2}} k_{0}|x|\right)\right],
\end{aligned}
$$

where

$$
E_{1}=\overrightarrow{\boldsymbol{\epsilon}} \cdot \overrightarrow{\boldsymbol{\epsilon}}_{0}, \quad E_{2}=(\overrightarrow{\boldsymbol{\epsilon}} \cdot \hat{b})\left(\vec{\epsilon}_{0} \cdot \hat{b}\right) .
$$

This quantity, once properly normalized, describes the quantum mechanical probability of measuring the polarization represented by the vector $\epsilon$ at a distance $|x|$ from the origin, where it was created with a polarization represented by $\epsilon_{0}$. Since we restrict ourselves to the case $\vec{k} \cdot \vec{B}=0$ and assume that the polarization vectors are orthogonal to the direction of propagation, we can write

$$
\begin{aligned}
\hat{k} & =\hat{x}, \quad \hat{b}=\hat{y}, \quad \vec{\epsilon}=\cos \alpha \hat{y}+\sin \alpha \hat{z}, \\
\vec{\epsilon}_{0} & =\cos \beta \hat{y}+\sin \beta \hat{z},
\end{aligned}
$$

so that

$$
E_{1}=\cos (\alpha-\beta), \quad E_{2}=\cos \alpha \cos \beta
$$

The extrema of Eq. (67), for a given initial angle $\beta$, are at

$$
\tan 2 \alpha(x)=\frac{[1+2 f(x)] \sin 2 \beta}{4 f(x)+[1+4 f(x)] \cos 2 \beta},
$$

where

$$
f(x)=\sin ^{2}\left(\frac{\vec{b}^{2}}{4 m^{2}} k_{0}|x|\right)
$$

corresponding to the values of the angle where the probability of finding the polarization vector is maximum or minimum. The mean value of the angle is

$$
\bar{\alpha}(x)=-\frac{1}{2} \frac{[1+2 f(x)] \sin 2 \beta}{[1+4 f(x)]+4 f(x) \cos 2 \beta} .
$$

If the electric field is initially parallel to the magnetic field, it remains parallel, i.e. $\alpha(x)=0$. Otherwise, a rotation in the plane of polarization appears.

The parameter characterizing the evolution is $k_{0}|x| \vec{b}^{2} / 2 m^{2}$. Usually, [13] mixing is treated via the classical evolution equation

$$
\left[\omega^{2}+\partial_{x}^{2}+\left(\begin{array}{ccc}
0 & 0 & 0 \\
0 & 0 & \omega b \\
0 & \omega b & -m^{2}
\end{array}\right)\right]\left(\begin{array}{c}
\omega \epsilon_{\perp} \\
\omega \epsilon_{\|} \\
a
\end{array}\right)=0 .
$$

Note that the contribution from the Euler-Heisenberg Lagrangian induced by the virtual contribution of electrons [14] has not been included. It is not difficult to verify that both methods lead to the same dispersion relations in the case where $\eta_{0}=0$.

\section{INFLUENCE OF THE AXION BACKGROUND}

Now, we return to the case with the axion background. In the limit where there is no magnetic field, we recover the pole structure already discussed in the first section, $\omega_{ \pm}^{2}=\vec{k}^{2} \pm\left|\eta_{0}\right||\vec{k}|$, whose implications in an astrophysical context were discussed in Refs. [9-11].

When the magnetic field is present, in addition to these poles, we have three additional poles manifest in the third term in Eq. (52), which in the limit $\eta_{0} \rightarrow 0$, correspond to the ones described following Eq. (53). Taking into account that $\eta_{0}$ is a very small quantity, we shall disregard terms proportional to $\eta_{0}^{2}$ in what follows. Then, these three poles exactly agree with the ones discussed in the previous section.

It is certainly of interest to consider how the simultaneous presence of the magnetic field and the cold axion background influences the kinematics. If we consider the processes $e \rightarrow e \gamma$ or $p \rightarrow p \gamma$ discussed in the introduction, in the presence of both the cold axion background and the magnetic field, the kinematical restrictions change. We defer this analysis to a separate publication.

In order to consider the rotation of the polarization plane, we note once again that $\eta_{0}$ is a rather small parameter. We shall neglect in the propagator all terms quadratic in $\eta_{0}$; then Eq. (52) becomes

$$
\begin{aligned}
\mathcal{D}_{\mu \nu}(k)= & \frac{-i g_{\mu \nu}}{k^{2}}+\frac{i k_{0}^{2} b_{\mu} b_{\nu}}{k^{2}\left[k^{2}\left(k^{2}-m^{2}\right)-k_{0}^{2} \vec{b}^{2}\right]} \\
& -g_{\mu}^{j} g_{\nu}^{l} \frac{\eta_{0} k_{0}^{2}\left[b_{j}(\vec{b} \times \vec{k})_{l}-b_{l}(\vec{b} \times \vec{k})_{j}\right]}{k^{4}\left[k^{2}\left(k^{2}-m^{2}\right)-k_{0}^{2} \vec{b}^{2}\right]} .
\end{aligned}
$$

Now, we contract with the polarization vectors: 


$$
\begin{aligned}
\epsilon^{\mu} \mathcal{D}_{\mu \nu}(k) \epsilon_{0}^{\nu}= & \frac{i E_{1}}{k^{2}}+\frac{i E_{2} k_{0}^{2} \vec{b}^{2}}{k^{2}\left[k^{2}\left(k^{2}-m^{2}\right)-k_{0}^{2} \vec{b}^{2}\right]} \\
& -\frac{E_{3} \eta_{0} k_{0}^{2} \vec{b}^{2} k_{1}}{k^{4}\left[k^{2}\left(k^{2}-m^{2}\right)-k_{0}^{2} \vec{b}^{2}\right]},
\end{aligned}
$$

where $E_{1}$ and $E_{2}$ are given in Eqs. (68) and (70), and

$$
\begin{aligned}
E_{3} & =(\vec{\epsilon} \cdot \hat{b})\left[\vec{\epsilon}_{0} \cdot(\hat{b} \times \hat{k})\right]-\left(\vec{\epsilon}_{0} \cdot \hat{b}\right)[\vec{\epsilon} \cdot(\hat{b} \times \hat{k})] \\
& =\sin (\alpha-\beta) .
\end{aligned}
$$

We implement for the piece proportional to $\eta_{0}$ a decomposition similar to the one described in the previous section:

$$
\begin{aligned}
& \frac{1}{k^{4}\left[k^{2}\left(k^{2}-m^{2}\right)-k_{0}^{2} \vec{b}^{2}\right]} \\
& \quad=\frac{\tilde{A}}{\vec{k}^{2}-k_{0}^{2}}+\frac{\tilde{B}}{\vec{k}^{2}-F}+\frac{\tilde{C}}{\vec{k}^{2}-G}+\frac{\tilde{D}}{\left(\vec{k}^{2}-k_{0}^{2}\right)^{2}} .
\end{aligned}
$$

$F$ and $G$ have been derived before. The new (tilded) coefficients are

$$
\begin{gathered}
\tilde{B}=\frac{1}{\left(k_{0}^{2}-F\right)^{2}(F-G)} \approx \frac{m^{2}}{k_{0}^{4} \vec{b}^{4}}, \\
\tilde{C}=-\frac{1}{\left(k_{0}^{2}-G\right)(F-G)} \approx \frac{1}{m^{6}}, \\
\tilde{D}=\frac{1}{\left(k_{0}^{2}-F\right)\left(k_{0}^{2}-G\right)} \approx-\frac{1}{k_{0}^{2} \vec{b}^{2}}, \\
\tilde{A}=-\tilde{B}-\tilde{C} \approx-\frac{m^{2}}{k_{0}^{4} \vec{b}^{4}} .
\end{gathered}
$$

We will again consider the propagation of an electromagnetic plane wave of frequency $\omega=k_{0}$ in the $\hat{x}$ direction, perpendicular to the magnetic field. We have

$$
\begin{aligned}
\epsilon^{\mu} & \mathcal{D}_{\mu \nu}\left(k_{0}, k_{1}\right) \epsilon_{0}^{\nu} \\
& =\frac{-i E_{1}}{k_{1}^{2}-k_{0}^{2}}+i E_{2} k_{0}^{2} \vec{b}^{2}\left(\frac{A}{k_{1}^{2}-k_{0}^{2}}+\frac{B}{k_{1}^{2}-F}+\frac{C}{k_{1}^{2}-G}\right) \\
& -E_{3} \eta_{0} k_{0}^{2} \vec{b}^{2}\left(\frac{\tilde{A} k_{1}}{k_{1}^{2}-k_{0}^{2}}+\frac{\tilde{B} k_{1}}{k_{1}^{2}-F}+\frac{\tilde{C} k_{1}}{k_{1}^{2}-G}+\frac{\tilde{D} k_{1}}{\left(k_{1}^{2}-k_{0}^{2}\right)^{2}}\right) .
\end{aligned}
$$

Then,

$$
\begin{aligned}
& \epsilon^{\mu} \mathcal{D}_{\mu \nu}\left(k_{0}, x\right) \epsilon_{0}^{\nu} \\
& \approx \\
& \approx \\
& \quad \frac{E_{1}}{2 k_{0}} e^{i k_{0}|x|}+E_{2} \frac{e^{i k_{0}|x|}}{2 k_{0}}\left(1-e^{i\left(\vec{b}^{2} / 2 m^{2}\right) k_{0}|x|}\right) \\
& \quad+i E_{3} \frac{\eta_{0} m^{2}}{2 k_{0}^{2} \vec{b}^{2}} e^{i k_{0}|x|}\left(1-e^{i\left(\vec{b}^{2} / 2 m^{2}\right) k_{0}|x|}-i \frac{k_{0}|x| \vec{b}^{2}}{m^{2}}\right) .
\end{aligned}
$$

For small values of $k_{0}|x| \vec{b}^{2} / m^{2}$, its square reduces to

$$
\begin{aligned}
& \left|\epsilon^{\mu} \mathcal{D}_{\mu \nu}\left(k_{0}, x\right) \epsilon_{0}^{\nu}\right|^{2} \\
& \quad \approx \frac{1}{4 k_{0}^{2}}\left[E_{1}^{2}+\left(\frac{\vec{b}^{2}}{2 m^{2}} k_{0}|x|\right)^{2}\left(E_{1} E_{2}+E_{2}^{2}\right)+3 \eta_{0}|x| E_{1} E_{3}\right],
\end{aligned}
$$

where terms of order $\eta_{0}^{2}$ have been neglected.

The extrema of Eq. (85) are at

$$
\tan 2 \alpha(x)=\frac{[1+2 f(x)] \sin 2 \beta+3 \eta_{0}|x| \cos 2 \beta}{4 f(x)+[1+4 f(x)] \cos 2 \beta-3 \eta_{0}|x| \sin 2 \beta},
$$

where

$$
f(x)=\frac{\vec{b}^{4}}{16 m^{4}} k_{0}^{2}|x|^{2},
$$

and the mean value of the angle

$$
\bar{\alpha}=-\frac{1}{2} \frac{[1+2 f(x)] \sin 2 \beta+3 \eta_{0}|x| \cos 2 \beta}{[1+4 f(x)]+4 f(x) \cos 2 \beta} .
$$

\section{PHYSICAL IMPLICATIONS}

In this paper, we have seen that high-energy charged particles moving in a spatially constant but time-varying axion background with frequency $m$ radiate at a rate that agrees with the one computed in Refs. [10,11], where the approximation $\omega, k \gg m$ was assumed. It was seen in Refs. $[10,11]$ that the effect increases as the wave number of the emitted photons decreases, and its possible detection (if at all) is likely to occur in the $\mathrm{MHz}$ range of radio waves. In this region, $k \ll m$ and, therefore, the heuristic arguments used in Refs. [10,11] could be questioned. The calculation presented here settles the issue. The effect under discussion is quite real and to the best of our knowledge would constitute the clearest (perhaps even the only ${ }^{4}$ ) observational evidence that axions or ALP constitute the bulk of the dark matter component of the Universe.

\footnotetext{
${ }^{4}$ A possible exception would be the confirmation of Ref. [15] that axions or ALP form Bose-Einstein condensates and caustics appear as a consequence in the galactic structures.
} 
The so-called direct observation experiments, such as ADMX, CAST or analogous ones may find evidence for the existence of a particle with the properties of the axion or ALP but this would not prove (although it would certainly be a tremendous hint) that axions or ALP constitute the missing mass of the Universe.

It is of course unfortunate that the amount of radiation predicted by the effect discussed here is very small; it is possibly within the sensitivity of long-wave radio antennae being commissioned or already deployed but around 6 orders of magnitude below the average value of the Galaxy synchrotron radiation background for the best value of $\eta_{0}$. The effect approximately scales as $\eta_{0}\left(\eta_{0} / k\right)^{1.5}$. We expect this parameter to be $\eta_{0}<$ $10^{-20} \mathrm{eV}$, given the current bound for $f_{a}$ and the matter density (assumed to be due to cold axions).

In principle, observations in regions of low magnetic field could increase the signal/background ratio by several orders of magnitude as the synchrotron radiation is proportional to $\vec{B}^{2}$, assuming that the flux of cosmic rays stays at the average value in galactic regions of low magnetic field. It should be noted that the assumption for the electron flux (electrons radiate most in the present mechanism [10]) was taken very conservatively to be the value measured by satellites, likely to be a gross underestimate of the value in inner parts of the Galaxy. On the contrary, the background quoted is the observed value. In view of these considerations, we believe that is important to refine the estimates before concluding whether this axion-induced bremsstrahlung could be measurable or not, or used to place relevant bounds for $\eta_{0}$ and hence on $f_{a}$. Note that photons radiated via this effect are circularly polarized, while synchrotron light is polarized in the plane of motion; measuring polarization may therefore help in the detection of the effect.

In any case, it should be said that the effects under discussion could be considerably enhanced for ALP models (assuming that the corresponding ALP particle condensates similarly to axions proper) because some constraints are evaded by these models. This is certainly something to have in mind and worthy of further investigation.

Another remarkable consequence of the presence of a slowly varying axion or ALP background is the fact that some wavelengths (actually very narrow bands, see Fig. 1 and its caption) are forbidden in the Universe (or at least where there are substantial concentrations of cold dark matter, if this is constituted by cold axions or ALP). This opens of course a door for another line of experiments that could potentially probe these forbidden wavelengths. The viability of these experiments, which appear very difficult unless the axion or ALP mass is known beforehand, deserves further investigation too.

We have also studied the effect on the polarization of photons propagating in this oscillatory pseudoscalar background. We assume that $\eta(t)=\eta_{0}$ provides a good guidance. The results presented here have to be considered as exploratory, and a more detailed account will be presented elsewhere. The relevant quantity that governs the change in the plane of polarization is the ratio $\omega x \vec{b}^{2} / 2 \mathrm{~m}^{2}$. The value of $|\vec{b}|$ ranges from $10^{-15} \mathrm{eV}$ for magnetic fields of $10 \mathrm{~T}$ (such as the ones employed in CAST) to $10^{-6} \mathrm{eV}$ for magnetar-strength fields, assuming that $f_{a} \sim 10^{7} \mathrm{GeV}$. Taking $m \sim 0.1 \mathrm{eV}$ as a reference value for the axion mass, this corresponds to the following approximate range:

$$
10^{-28}<\frac{\vec{b}^{2}}{m^{2}}<10^{-10}
$$

We qualitatively reproduce previous results [13] in the case where only the magnetic field is considered. However, since we have derived the complete quantum propagator when photons propagate through an oscillating cold axion coherent background, we can examine the modifications due to it. We find that, quite remarkably, the modification in the ellipticity is independent of the light wavelength and also of the axion mass. It is probably even more notable that it is also independent of the magnetic field itself, even if one needs to introduce one to begin with. We should warn the reader that because the results on the polarization are derived for constant $\eta$, they are strictly valid for very short distances $(<2 \pi / m)$ only. The astrophysical consequences of the results presented here are yet to be fully explored. Note that to provide more realistic results, other medium effects (such as an effective photon mass or the Euler-Heisenberg effective Lagrangian) should be considered, too.

Clearly, the presence of the cold axion background modifies the properties of photon propagation in rather interesting ways. The modifications are tiny, but some of them may perhaps be experimentally or observationally explored. This could possibly shed some light on the nature of dark matter.

\section{ACKNOWLEDGMENTS}

We acknowledge the financial support of MICINN through projects FPA2010-20807, Consolider CPAN, and the support of SRG2009502. We thank A. Andrianov and F. Mescia for numerous discussions. We would also like to thank K. Zioutas for encouraging us to examine in detail the effect of magnetic fields. 
[1] R. D. Peccei and H. R. Quinn, Phys. Rev. Lett. 38, 1440 (1977); S. Weinberg, Phys. Rev. Lett. 40, 223 (1978); F. Wilzcek, Phys. Rev. Lett. 40, 279 (1978).

[2] L. Abbott and P. Sikivie, Phys. Lett. B 120, 133 (1983); Axions: Theory, Cosmology and Experimental Searches, edited by M. Kuster, G. Raffelt and B. Beltran, Lecture Notes in Physics Vol. 741 (Springer-Verlag, Heidelberg, Germany, 2008).

[3] R. Battesti et al., Lect. Notes Phys. 741, 199 (2008); P. Sikivie, Phys. Rev. Lett. 51, 1415 (1983); 52, 695 (1984); D. Dicus, E. Kolb, V. Teplitz, and R. Wagoner, Phys. Rev. D 18, 1829 (1978); G. Raffelt, Phys. Rev. D 33, 897 (1986); D. Lazarus et al., Phys. Rev. Lett. 69, 2333 (1992); Y. Inoue et al., Phys. Lett. B 668, 93 (2008); G. Raffelt, Lect. Notes Phys. 741, 51 (2008).

[4] K. Nakamura et al. (Particle Data Group), J. Phys. G 37, 075021 (2010).

[5] F. Bergsma et al. (CHARM Collaboration), Phys. Lett. B 157, 458 (1985).

[6] J. Jaeckel, E. Masso, J. Redondo, A. Ringwald, and F. Takahashi, Phys. Rev. D 75, 013004 (2007).

[7] E. Arik et al. (CAST collaboration), J. Cosmol. Astropart. Phys. 02 (2009) 008.

[8] M. Dine, W. Fischler, and M. Srednicki, Phys. Lett. B 104, 199 (1981); A. R. Zhitnitsky, Sov. J. Nucl. Phys. 31, 260
(1980); J. E. Kim, Phys. Rev. Lett. 43, 103 (1979); M. A. Shifman, A. I. Vainshtein, and V. I. Zakharov, Nucl. Phys. B 166, 493 (1980).

[9] A. A. Andrianov, D. Espriu, P. Giacconi, and R. Soldati, J. High Energy Phys. 09 (2009) 057.

[10] D. Espriu, F. Mescia, and A. Renau, J. Cosmol. Astropart. Phys. 08002 (2011).

[11] A. Andrianov, D. Espriu, F. Mescia, and A. Renau, Phys. Lett. B 684, 101 (2010).

[12] R. de L. Kronig and W. G. Penney, Proc. R. Soc. A 130, 499 (1931).

[13] G. Raffelt and L. Stodolsky, Phys. Rev. D 37, 1237 (1988).

[14] S. L. Adler, Ann. Phys. (Leipzig) 67, 599 (1971); Z. Bialynicka-Birula and I. Bialynicki-Birula, Phys. Rev. D 2, 2341 (1970); J. S. Heyl and L. Hernquist, J. Phys. A 30, 6485 (1997); W. Dittrich and H. Gies, in Frontier Tests of QED and Physics of the Vacuum, Sandansky, Bulgaria, 1998, (Heron Press, Birmingham, United Kingdom, 1998); H. Gies, in Quantum Electrodynamics and Physics of the Vacuum: QED 2000, Trieste, Italy, 2000 (Springer-Verlag, New York, 2011; V. A. De Lorenci, R. Klippert, M. Novello, and J. M. Salim, Phys. Lett. B 482, 134 (2000).

[15] P. Sikivie, Phys. Lett. B567, 1 (2003). 